\title{
ARTICLES
}

\section{Analysis of Voltage-Gated Potassium Channel $\beta 1$ Subunits in the Porcine Neonatal Ductus Arteriosus}

\author{
EMIKO HAYAMA, SHIN-ICHIRO IMAMURA, CUIJIAO WU, MAKOTO NAKAZAWA, RUMIKO MATSUOKA, \\ AND TOSHIO NAKANISHI \\ Department of Pediatric Cardiology, The Heart Institute of Japan, Tokyo Women's Medical University, Shinjuku-ku, \\ Tokyo, 162-8666, Japan
}

\begin{abstract}
The voltage-gated potassium channels (Kv) are partially responsible for the contraction/relaxation of blood vessels in response to changes in the $\mathrm{PO}_{2}$ level. The present study determined the expression of $\mathrm{Kv} \beta 1$ and four oxygen-sensitive $\mathrm{Kv} \alpha$ subunits (Kv1.2, Kv1.5, Kv2.1, and Kv9.3) in the ductus arteriosus (DA), the aorta (Ao), and the pulmonary artery (PA) in porcine neonates immediately after birth. We cloned three $\operatorname{Kv} \beta 1$ transcript variants $(\mathrm{Kv} \beta 1.2, \operatorname{Kv} \beta 1.3$, and $\mathrm{Kv} \beta 1.4), \mathrm{Kv} 1.2, \mathrm{Kv} 1.5$, and $\mathrm{Kv} 9.3$ from piglets. Three $\mathrm{Kv} \beta 1$ transcripts, Kv1.2, Kv1.5, and Kv9.3, encode predicted proteins of 401, 408, 202, 499, 600, and 491 residues. These Kv showed a high degree of sequence conservation with the corresponding $\mathrm{Kv}$ in human. Northern and quantitative real-time PCR (qr-PCR) analyses showed that $\mathrm{Kv} \beta 1.2$ expression was high in the DA and Ao but low in the PA. Kv1.5 expression was high in the Ao and PA but low in the DA. Expression of $\operatorname{Kv} \beta 1.3, \operatorname{Kv} \beta 1.4$, $\mathrm{Kv} 1.2, \mathrm{Kv} 2.1$, and Kv9.3 was low in these blood vessels. The inactivation property of $\mathrm{Kv} \beta 1.2$ against $\mathrm{Kv} 1.5$ was confirmed using Xenopus laevis oocytes. Our findings suggest that the molecular basis for the differential electrophysiological characteristics including opposing response to oxygen in the DA and the PA are partially due to diversity in expression of $\mathrm{Kv} 1.5$ and $\mathrm{Kv} \beta 1.2$ subunits. The high expression of $\mathrm{Kv} \beta 1.2$ and relatively low expression of Kv1.5 in the DA might be partially responsible for the ductal closure after birth. (Pediatr Res 59: 167-174, 2006)
\end{abstract}

$\mathrm{T}$ DA plays a pivotal role in fetal development by diverting the passage of blood from the PA into the Ao (1). At birth, functional closure of the DA is initiated by an increase in $\mathrm{Po}_{2}$ (2-4). Previously, we reported that oxygen increases the intracellular calcium concentration and causes contraction of the DA (5). In that report, closure of the

Received February 2, 2005; accepted August 5, 2005.

Correspondence: Toshio Nakanishi, M.D., Ph.D., Department of Pediatric Cardiology, The Heart Institute of Japan, Tokyo Women's Medical University, 8-1 Kawada-cho, Shinjuku-ku, Tokyo, 162-8666, Japan; e-mail: pnakanis@hij.twmu.ac.jp

Supported by a grant-in-aid from the Japanese Ministry of Education, Culture, Sports, Science and Technology, by a grant-in-aid from the Japan Research Promotion Society for Cardiovascular Diseases and by a grant for the Promotion of the Advancement of Education and Research in Graduate Schools (2002-2005). Part of this work was supported by a grant for the Encouraging Development of Strategic Research Centers, Special Coordination Funds for Promoting Science and Technology, Ministry of Education, Culture, Sports, Science and Technology.

DOI: 10.1203/01.pdr.0000196736.89742.c1
ATP-sensitive potassium channel was suggested to be involved in the DA contraction due to depolarization of the membrane potential, which, in turn, increases calcium influx via the voltage-dependent calcium channel. However, mechanisms other than the ATP-sensitive potassium channel may also be involved in the oxygen-induced contraction and these remain to bestudied.

Although the mechanism has not been elucidated, $\mathrm{Kv}$ channels in SMC may also be involved in oxygen-induced vascular constriction (6). The oxygen sensitivity of $\mathrm{Kv}$ has been studied extensively, and Kv1.2, Kv1.5, Kv2.1, Kv3.1b, Kv4.2, Kv4.3, and Kv9.3 are major candidates for the oxygen-sensitive potassium channel (7-13). Among them, it was reported that SMC in the DA expresses Kv1.5 and Kv2.1 (14).

Functional $\mathrm{Kv}$ are composed of a tetramer of $\mathrm{Kv} \alpha$ subunits (15), which co-assemble with $\operatorname{Kv} \beta$ subunits $(16-21)$. The $\operatorname{Kv} \beta$ subunits have variants known as $\operatorname{Kv} \beta 1.1, \operatorname{Kv} \beta 1.2$, and $\operatorname{Kv} \beta 1.3(22,23), \operatorname{Kv} \beta 2.1$ and $\operatorname{Kv} \beta 2.2(23), \operatorname{Kv} \beta 3$ (24), and $\operatorname{Kv} \beta 4.1$ (25), which show distinct modulatory effects on the $\mathrm{Kv} \alpha$ subunits $(16,18) . \mathrm{Kv} \beta 1.2$ is a potent blocker for $\mathrm{Kv} 1.1$,

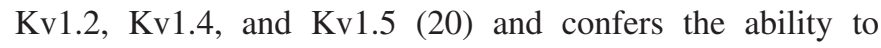
respond to hypoxia to Kv4.2 (12).

The interaction of the $\operatorname{Kv} \beta 1$ subunits with $\alpha$ subunits is consistent with the $\alpha_{4} \beta_{\mathrm{n}}$ model, where n equals $0,1,2,3$, or 4 , depending upon the relative concentration of $\alpha$ and $\beta$ subunits. The $\alpha_{4} \beta_{\mathrm{n}}$ stoichiometry allows for gradual changes in $\mathrm{Kv} \beta 1$-mediated inactivation (19).

Thus, oxygen-sensitive $\mathrm{Kv} \alpha$ subunits are possibly regulated by $\operatorname{Kv} \beta$ subunits based on the relative expression levels. Therefore, we hypothesized that diversity in the expression of oxygen-sensitive $\operatorname{Kv} \alpha$ or $\operatorname{Kv} \beta$ subunits in the DA as well as in the contiguous Ao and PA might be responsible for various responses to change in $\mathrm{Po}_{2}$ at birth.

\footnotetext{
Abbreviations: Ao, aorta; b-PA, third and fourth branched pulmonary artery; DA, ductus arteriosus; EC, endothelial cells; Kv, voltage-gated potassium channel; PA, pulmonary artery; SkM, skeletal muscle; SMC, smooth muscle cells
} 


\section{METHODS}

Animals and tissue samples. We chose the pig model for our studies to obtain enough starting blood vessel specimens. Various tissues were taken from newborn piglets of the Landrace/Large White/Duroc composite killed with chloroform immediately after birth. The adventitia and the endothelial layer of the DA, Ao, and PA were removed in saline. All tissues were quickly frozen and stored at $-135^{\circ} \mathrm{C}$. Treatment of animals conformed to the guiding principles of the American Physiologic Society. The experiment was approved by the Ethical Committee of Animal Experiments of Tokyo Women's Medical University.

RNA isolation. Total RNA of DA, Ao, and PA were isolated from 14, 2, or 3 pooled segments, respectively. We followed the RNA extraction methods described by Chomczynski and Sacchi (26). To reduce the level of contamination with proteoglycans and polysaccharides, RNA extraction solution (27) was used to precipitate the RNA.

Cloning. A strategy combining a homology-based PCR and rapid amplification of cDNA ends (RACE) was used to identify $\mathrm{Kv} \beta 1, \mathrm{Kv} 1.2$, and $\mathrm{Kv} 1.5$ cDNA. Total RNA extracted from the DA, brain and SkM were reversetranscribed into cDNA using PowerScript Reverse Transcriptase (BD Biosciences Clontech, Palo Alto, CA). Based on the conserved regions of the human, mouse, and rat $\operatorname{Kv} \beta$ cDNA, we designed primers to amplify partial $\operatorname{Kv} \beta$ cDNA and to carry out RACE (Table 1 ). RACE was performed using a SMARTRACE cDNA amplification kit (BD Biosciences Clontech). Obtained cDNA were cloned into pT7Blue T-Vector (Novagen, Darmstadt, Germany), and sequenced with a BigDye terminator sequencing kit (Applied Biosystems, Foster City, CA) and a 3100 genetic analyzer (Applied Biosystems). Sequences were analyzed with SeqMan II (DNASTAR, Madison, WI) and GENETYX-MAC (Software Development, Tokyo, Japan).

A porcine lambda cDNA library, constructed from cultured smooth muscle thoracic aorta (Stratagene, La Jolla, CA), was screened using a probe prepared from rat pCIneo-Kv9.3 labeled with horseradish peroxidase using an enhanced chemiluminescence (ECL) direct nucleic-acid labeling system (Amersham Pharmacia Biotech UK, Ltd., Little Chalfont, Buckinghamshire, UK). Positive phages were located with ECL detection reagents (Amersham Pharmacia Biotech UK, Ltd.).

Porcine genomic DNA was extracted from the kidney with a DNeasy Tissue kit (QIAGEN, Hilden, Germany), and used as a template to obtain the PCR products containing the $5^{\prime}$-end region of the $\operatorname{Kv} \beta 1.4$ cDNA, using a DNA Walking SpeedUp Premix kit (Seegene, Seoul, Korea) with three target-specific primers.
Northern blot analysis. Northern blot analysis was performed as described previously with modification (28). Isolated total RNA (20 $\mu$ g/lane) was electrophoresed on denaturing $1.0 \%$ agarose-formaldehyde gels and transferred to nitrocellulose membranes (NitroPure, GE Osmonics, Trevose, PA). cDNA probes were generated using either restriction enzyme digestion or PCR followed by gel-purification. A $\operatorname{Kv} \beta 1$ common probe (1050 bp), corresponding to almost all the outermost half of the $3^{\prime}$-untranslated region (UTR), was prepared by digestion of the $\operatorname{Kv} \beta 13^{\prime}$-RACE clone with HindIII (in the $3^{\prime}$-UTR) and SalI (in the multiple cloning site). A Kv $\beta 1$.2-specific probe (170 bp), corresponding to nucleotides $67-236$ of the porcine $\mathrm{Kv} \beta 1.2$, and a $\mathrm{Kv} \beta 1.3$-specific probe (1094 bp), corresponding to nucleotides -875 to 219 of the porcine $\operatorname{Kv} \beta 1.3$, were generated using PCR. Kv1.2, Kv1.5, and Kv9.3 probes (710, 804, and $1476 \mathrm{bp}$ ), corresponding to nucleotides 1352-2061 of the porcine Kv1.2, nucleotides 1737-2541 of the porcine Kv1.5, and nucleotides $1-1476$ of the porcine Kv9.3, were generated using PCR. A glyceraldehyde 3-phosphate dehydrogenase (GAPDH) probe ( $682 \mathrm{bp})$, corresponding to nucleotides 549-1230 of the porcine GAPDH (GenBank accession number AF017079), was also generated using PCR. The cDNA probes were labeled using a Nick translation kit (Roche Diagnostics, Mannheim, Germany) with $\left[\alpha{ }^{32} \mathrm{P}\right] \mathrm{dCTP}$. Hybridization was performed overnight at $65^{\circ} \mathrm{C}$. The blot was washed with $1 \times$ SSC and $0.1 \%$ SDS and visualized with an image analyzer BAS 2000 (Fuji Photo Film, Tokyo, Japan).

qr-PCR. DNase I digestion of total RNA was carried out on an RNeasy Mini Kit (QIAGEN) extraction column using an RNase-Free DNase Set (QIAGEN). The total RNA was reverse-transcribed into cDNA with random hexamers and MultiScribe Reverse Transcriptase (Applied Biosystems). As a control for genomic DNA contamination, all cDNA synthesis reactions were set up with additional samples lacking reverse transcriptase.

We designed all primers and probes used for qr-PCR using Primer Express software (Applied Biosystems) (Table 2). The $25-\mu \mathrm{L}$ qr-PCR reaction included $1 \times$ Premix Ex Taq ${ }^{\mathrm{TM}}$ (TAKARA BIO, Shiga, Japan), $900 \mathrm{nM}$ of the respective primers, $250 \mathrm{nM}$ of the respective probe, $1 \times$ ROX reference dye, and template cDNA corresponding to $5 \mathrm{ng}$ total RNA. 18S ribosomal RNA (Applied Biosystems) was used as an internal control. For standard curve production, the plasmids harboring the corresponding $\mathrm{Kv}, \mathrm{EC}$-specific marker, SMC-specific marker, and ribosomal 18S cDNA fragments were linearized by appropriate restriction enzyme digestion, purified, quantified, and diluted serially in water containing yeast tRNA $(50 \mathrm{ng} / \mu \mathrm{L})$. The second passage of primary culture of porcine aortic EC (Cell Applications, San Diego, CA) and the first passage of primary culture of porcine aortic SMC (Cell Applications) were used as positive controls to estimate potential

Table 1. Sequence of primers used in the clonings

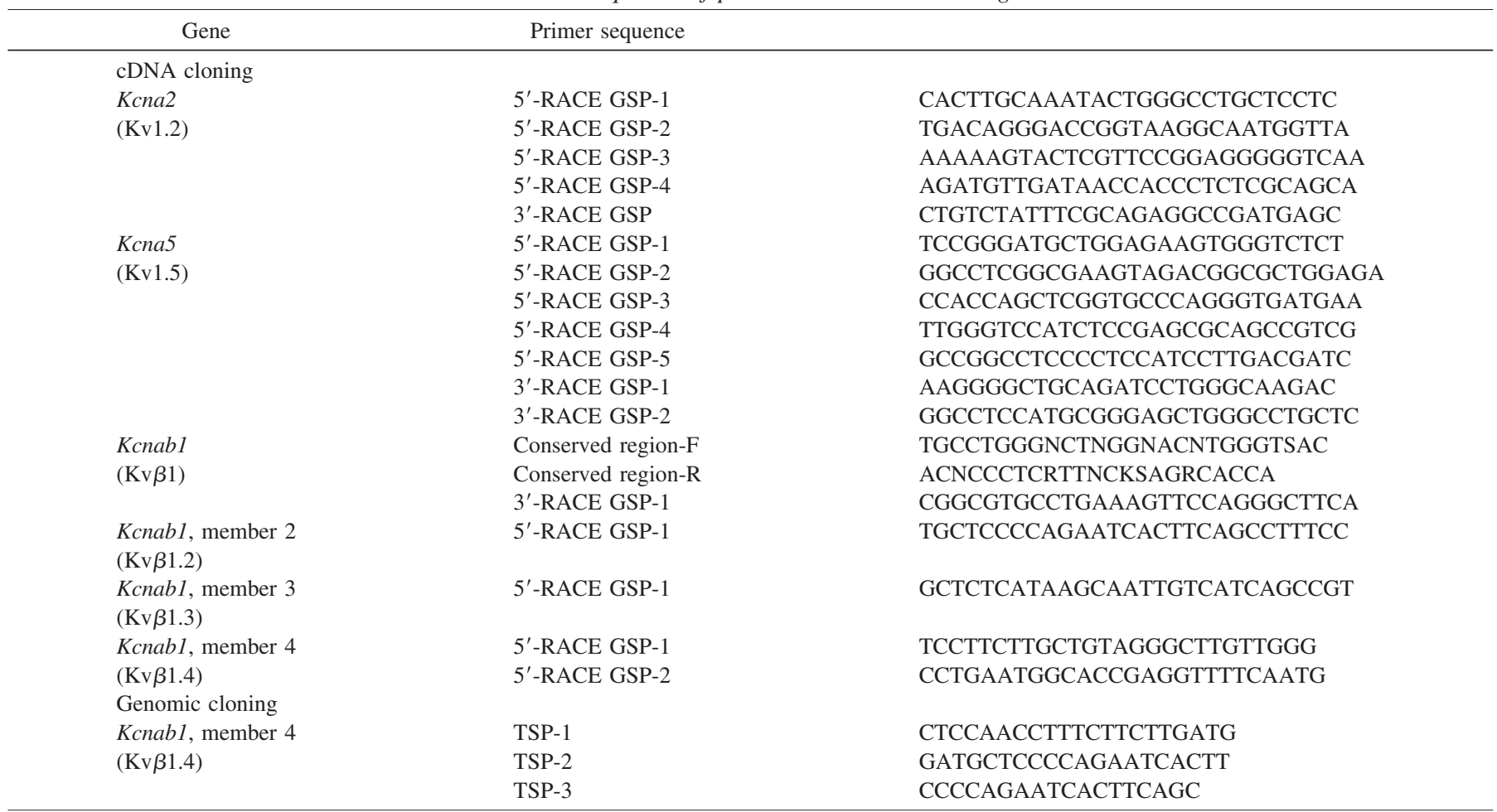

GSP, gene-specific primer; TSP, target-specific primer. 
Table 2. Sequences of primers and probes used in the real-time PCR experiment

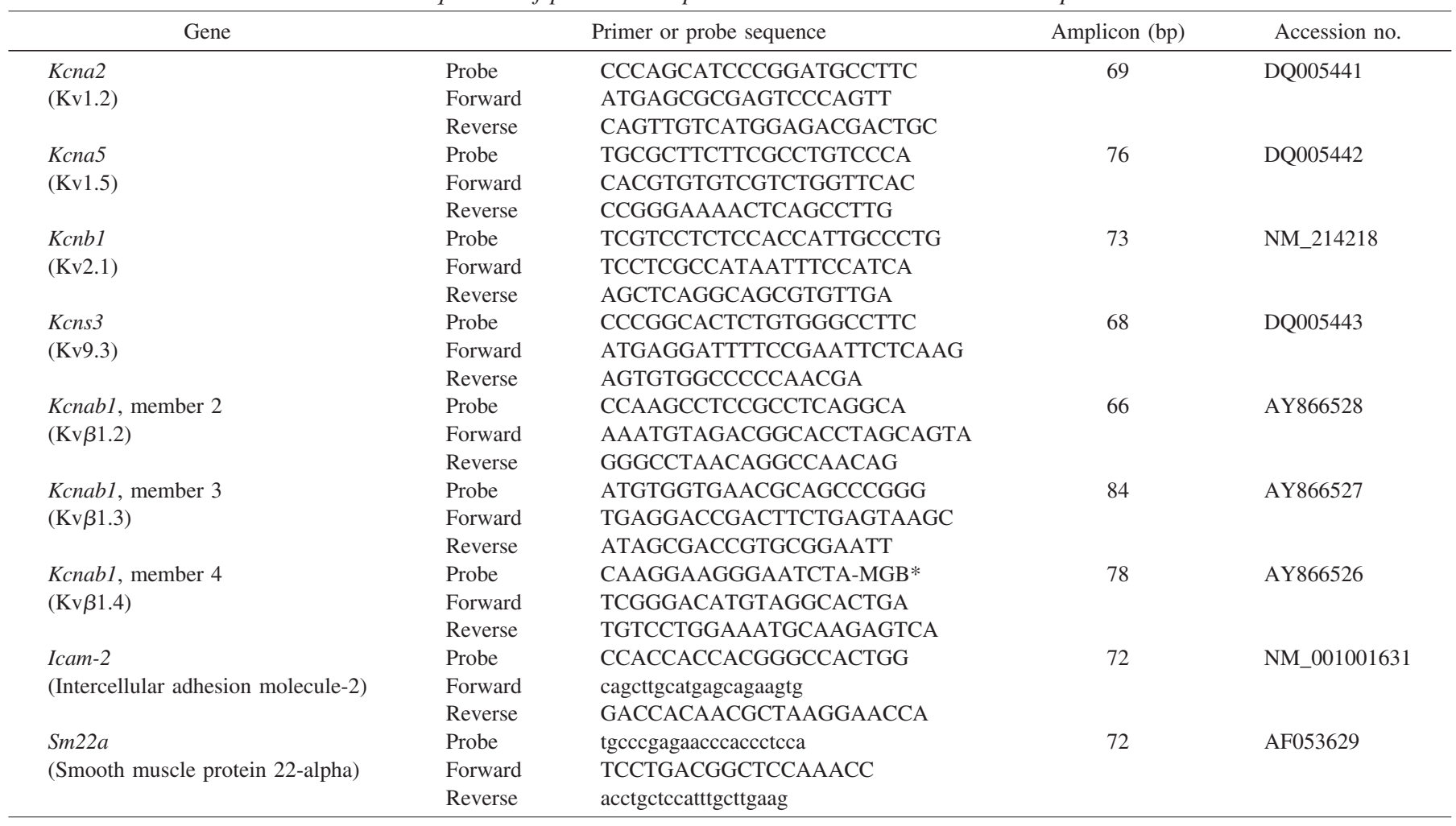

* Minor Groove Binder.

contribution of EC. Real-time PCR of standards and internal controls was performed each time on the same 96-well plate with the samples being quantified.

To quantify target mRNA expression, cDNA copy numbers were calculated based on the results of the standard curve of the same run. The correlation coefficients were always above 0.98 . Each sample was analyzed in triplicate, and arithmetic means were calculated. The cDNA copy numbers were then normalized using the calculated $18 \mathrm{~S}$ cDNA copy number of the same sample.

cRNA synthesis, injection, and electrophysiological measurement in Xenopus oocytes. Capped cRNA were synthesized in vitro from the linearized plasmids harboring the coding sequences of Kv1.5 or Kv $\beta 1.2$ using T7 RNA polymerase (Promega, Madison, WI). Defolliculated Xenopus laevis oocytes were prepared, as described previously (29), and injected with $50 \mathrm{~nL}$ of cRNA. A single oocyte was impaled with two standard glass microelectrodes (1-2.5-megohm resistance) filled with $3 \mathrm{M} \mathrm{KCl}$ and maintained under a voltage clamp using a Gene Clamp 500B amplifier (Axon Instruments, Burlingame, CA). Stimulation of the preparation, data acquisition, and analysis were performed using pClamp software (Clampex 8.2 and Clampfit 8.2, Axon Instruments). All experiments were performed at room temperature in ND96 solutions containing $96 \mathrm{mM} \mathrm{NaCl}, 2 \mathrm{mM} \mathrm{KCl}, 1.8 \mathrm{mM} \mathrm{CaCl}_{2}, 2 \mathrm{mM}$ $\mathrm{MgCl}_{2}$, and $5 \mathrm{mM}$ HEPES at $\mathrm{pH}$ 7.4.

\section{RESULTS}

Cloning. The structures of the three porcine $\operatorname{Kv} \beta 1$ subunit nucleotide sequences were aligned with the human $\operatorname{Kv} \beta 1$ subunit (Fig. 1A). Predicted amino acid sequences of porcine $\operatorname{Kv} \beta 1$ variants are presented in Figure $1 B$. Two porcine $\operatorname{Kv} \beta 1$ subunits cloned from DA and brain encoded the predicted proteins of 408 and 401 amino acids that share a high degree of sequence conservation with $\operatorname{Kv} \beta 1.2(97.8 \%)$ and $\operatorname{Kv} \beta 1.3$ (99.5\%) in human, respectively. Over the C-terminal 328 amino-acids, they were identical and $99.4 \%$ identical to hu$\operatorname{man} \operatorname{Kv} \beta 1 . \operatorname{Kv} \beta 1.2$ and $\operatorname{Kv} \beta 1.3$ differed in length, 80 and 73 amino-acids, respectively, and in the sequence of their $\mathrm{N}$ - terminal regions. The N-terminal regions of porcine $\operatorname{Kv} \beta 1.2$ and $\operatorname{Kv} \beta 1.3$ shared an identity of $91.3 \%$ and $100 \%$ to the corresponding region of human $\operatorname{Kv} \beta 1.2$ and $\operatorname{Kv} \beta 1.3$.

The third porcine $\mathrm{Kv} \beta 1$ variant cloned from SkM contained 116 distinct nucleotides at the $5^{\prime}$-end and 823 nucleotides at the opposite end of the conserved C-terminal cDNA, but lacked 164 nucleotides near to the $5^{\prime}$-end of $\mathrm{C}$-terminal conserved cDNA and the entire cDNA encoding the variable $\mathrm{N}$-terminal regions. No translational start codon was present in the 116 distinct nucleotides. Taking the first in-frame ATG as the start codon and the stop codon as being similar to other $\mathrm{Kv} \beta 1$ subunits, an open reading frame of $609 \mathrm{bp}$ encoding a protein of 202 amino-acids, that would be completely identical with the latter half of the C-terminal region of other $\operatorname{Kv} \beta 1$ subunits, was predicted. The 116 distinct nucleotides at the $5^{\prime}$-end of this porcine $\operatorname{Kv} \beta 1$ subunit were not similar to the $\mathrm{N}$-terminal cDNA sequence of human $\operatorname{Kv} \beta 1.1$. This previously unrecognized $\operatorname{Kv} \beta 1$ subunit was designated $\operatorname{Kv} \beta 1.4$. Genomic sequencing of the 5 '-end region of $\operatorname{Kv} \beta 1.4$ revealed that this transcript retained a highly homologous sequence with the $3^{\prime}$-end region of human $\operatorname{Kv} \beta 1$ intron 4 (Fig. 2). (GenBank accession numbers: $\operatorname{Kv} \beta 1.2$ AY866528; $\operatorname{Kv} \beta 1.3$, AY866527; and $\mathrm{Kv} \beta 1.4$, AY866526)

Porcine Kv1.2 (Fig. 3A), Kv1.5 (Fig. 3B), and Kv9.3 (Fig. $3 C$ ) subunits cloned from the brain, DA, and Ao encoded the predicted proteins of 499, 600, and 491 amino-acids that share a high degree of sequence conservation with the homologous proteins, Kv1.2 (99.6\%), Kv1.5 (86.5\%), and Kv9.3 (96.3\%) in human, respectively (GenBank accession 
A

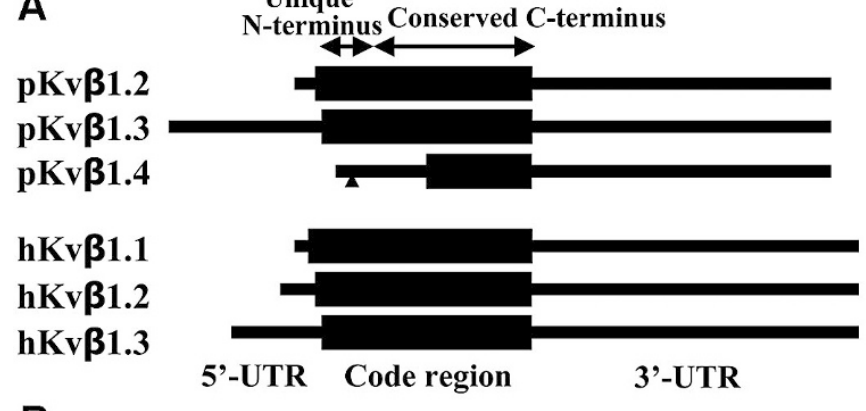

B

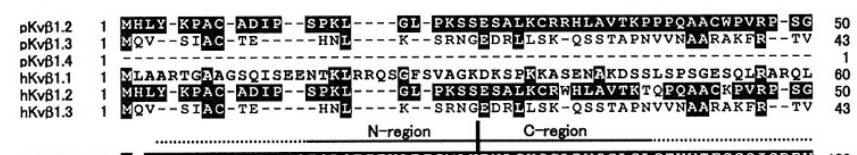

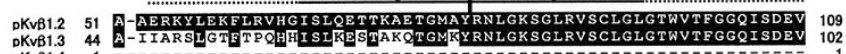

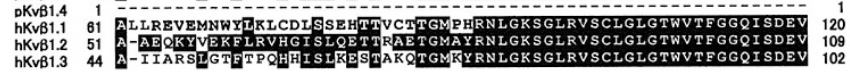

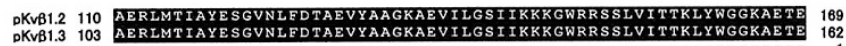

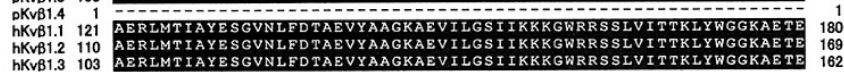

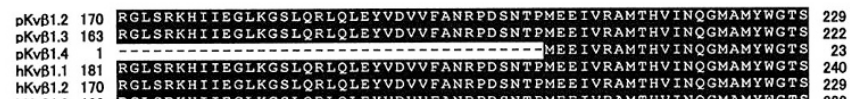

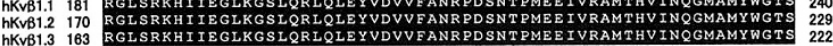

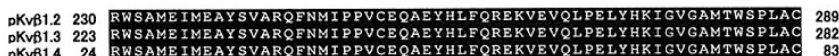

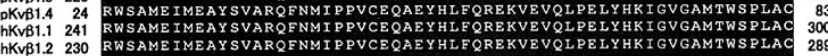

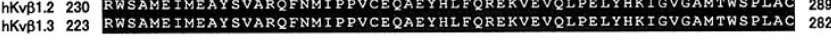

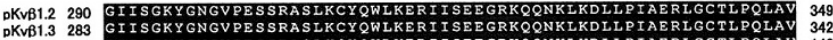

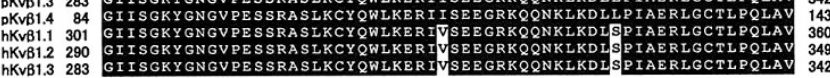

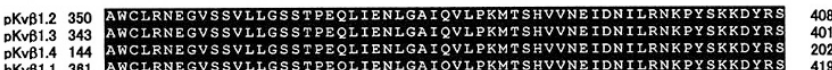

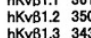

Figure 1. Schematic presentation of nucleotide sequences $(A)$ and predicted amino acid sequences $(B)$ of porcine and human $\operatorname{Kv} \beta 1$ transcripts. (A) Code regions are shown in thick lines and UTR regions in thinner lines. The closed triangle indicates a critical stop codon on a $\operatorname{Kv} \beta 1.4$ nucleotide sequence. Due to this stop codon, the $\operatorname{Kv} \beta 1.4$ transcript has a smaller code region than the other $\mathrm{Kv} \beta 1$ transcripts. p: porcine, h: human $(B) \mathrm{Kv} \beta 1$ amino acid sequences were deduced from the cloned $\operatorname{Kv} \beta 1.2, \operatorname{Kv} \beta 1.3$, and $\operatorname{Kv} \beta 1.4$ nucleotide sequences, and aligned with amino acid sequences of human $\operatorname{Kv} \beta 1$ transcript variants. Amino acid residues identical to the porcine $\operatorname{Kv} \beta 1.2$ are shaded.

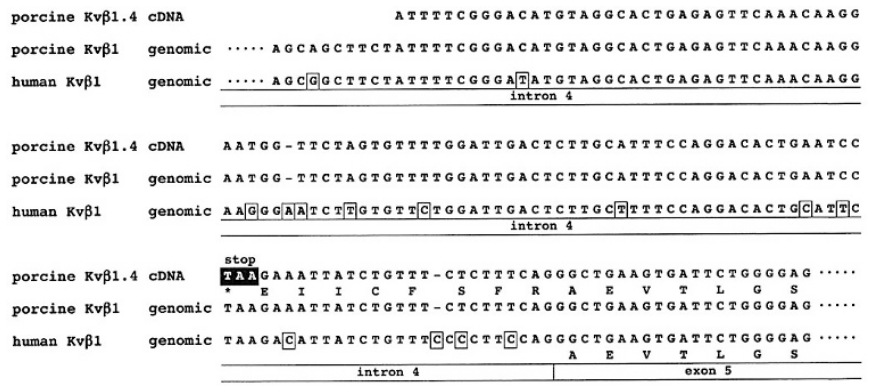

Figure 2. Comparison of $\mathrm{Kv} \beta 1.4$ partial cDNA, genomic DNA, and human genomic $\operatorname{Kv} \beta 1$ DNA. The 116 nucleotides at the $5^{\prime}$-end of the $\operatorname{Kv} \beta 1.4$ cDNA and the near 3 '-end of human intron 4 DNA sequences were aligned. The dashed box indicates a stop codon at the $5^{\prime}$-end of Kv $\beta 1.4$ cDNA. Open boxes denote nucleotides that are not conserved between porcine and human $\operatorname{Kv} \beta 1$.

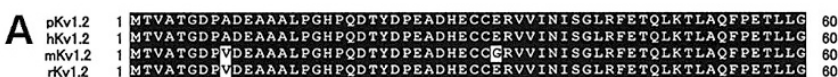

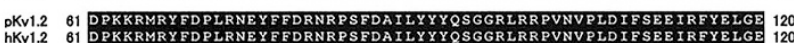

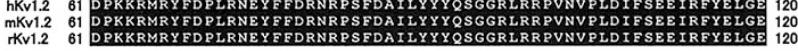

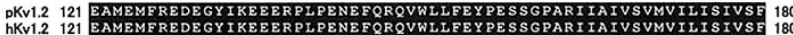

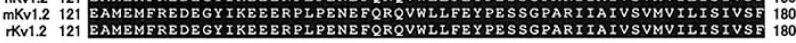

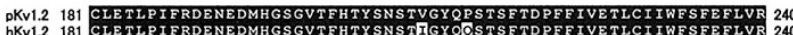

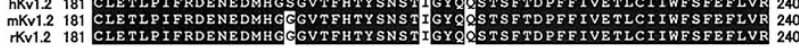

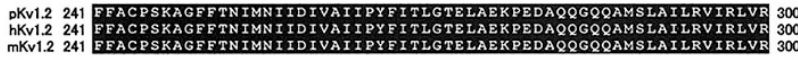

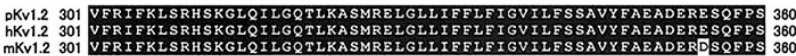

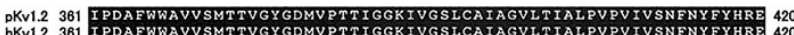

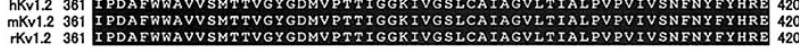

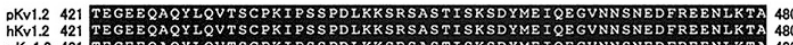

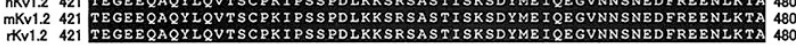

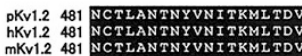

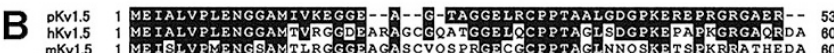

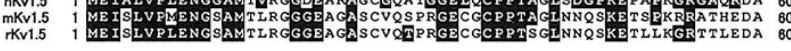

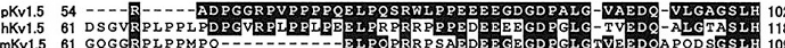

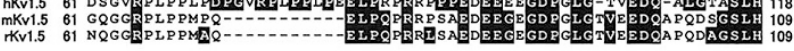

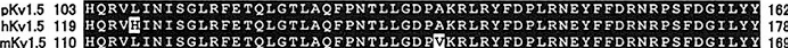

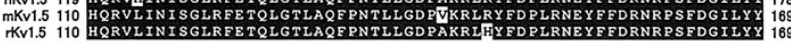

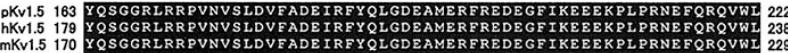

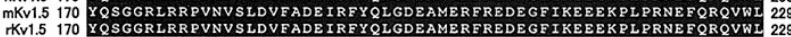

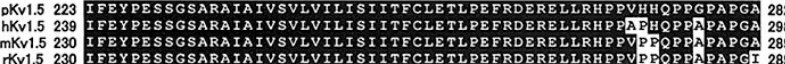

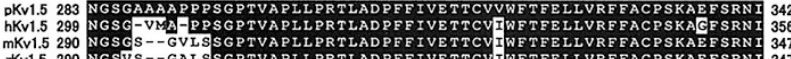

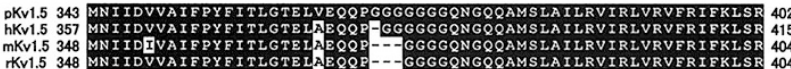

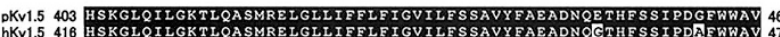

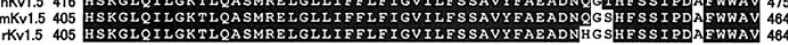

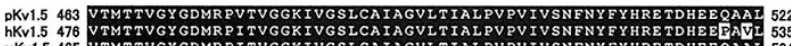

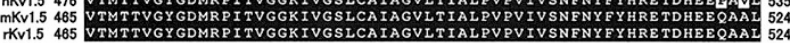

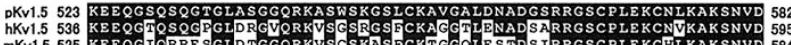

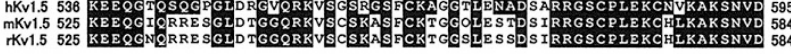

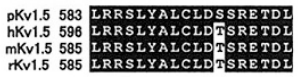

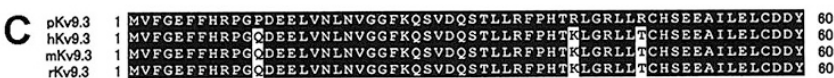

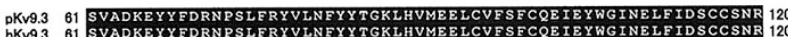

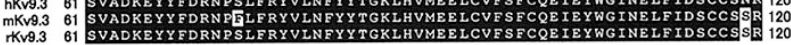

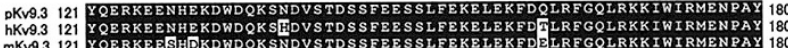

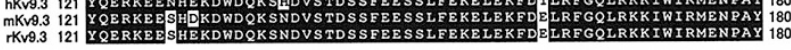

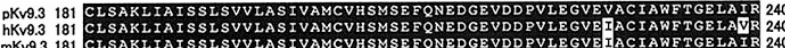

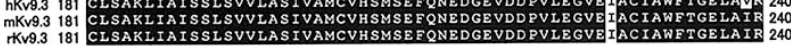

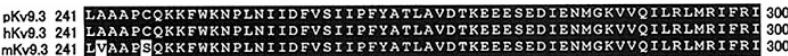

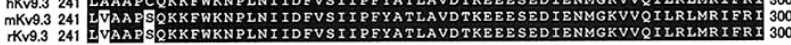

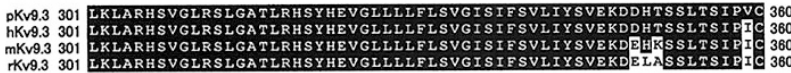

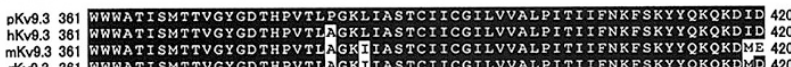

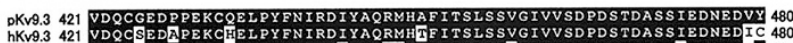

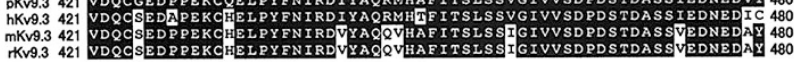
PKV9.3 481 NIASLENGTAF
hKV 9.3481 NTTSLENCTAF

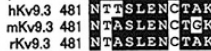



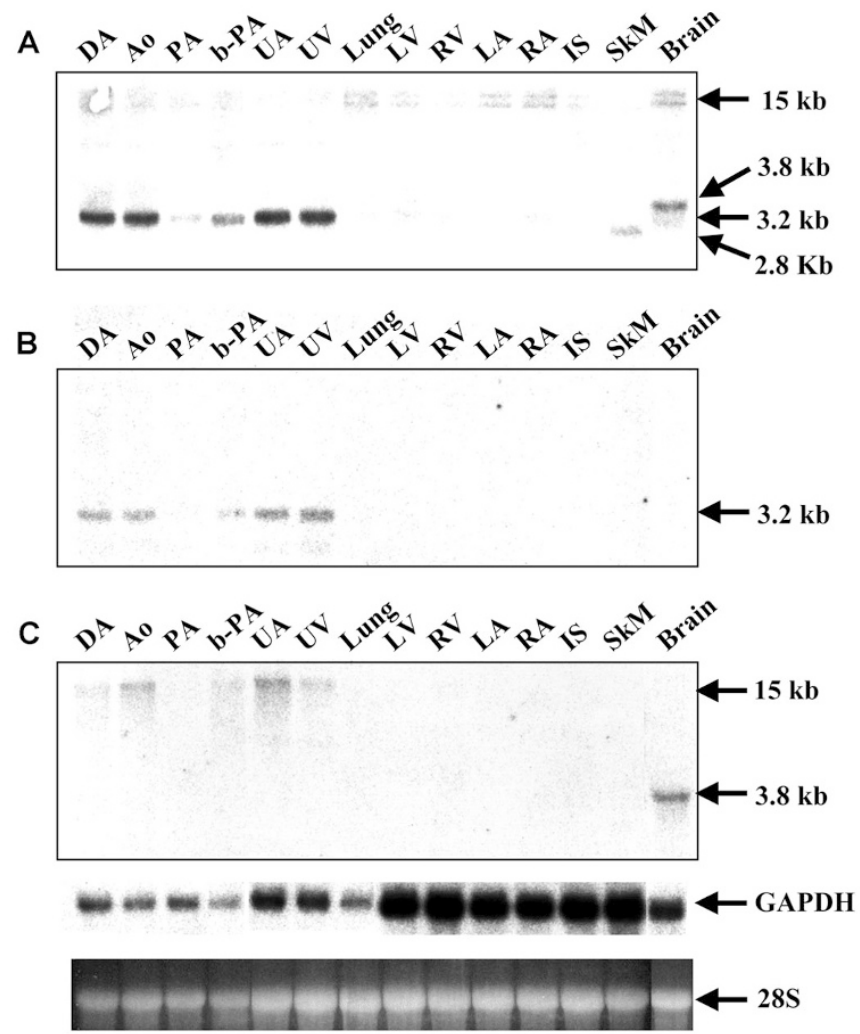

Figure 4. Northern blot analysis of $\operatorname{Kv} \beta 1$ subunits using $(A) \operatorname{Kv} \beta 1$ common, (B) $\mathrm{Kv} \beta 1.2-$, and $(C) \mathrm{Kv} \beta 1.3$-specific probes. (A) The $2.8 \mathrm{~kb}$ band was detected in the SkM. The $3.2 \mathrm{~kb}$ band was detected exclusively in the DA, Ao, $\mathrm{UA}$, and UV, weakly in the b-PA and faintly in the PA and brain. The $3.8 \mathrm{~kb}$ band was detected mainly in the brain. Multiple transcripts of approximately $15 \mathrm{~kb}$ were detected in many of the samples. $(B)$ The $\mathrm{Kv} \beta 1.2$-specific probe detected the $3.2 \mathrm{~kb}$ transcript in the DA, Ao, b-PA, UA, and UV. (C) The $\mathrm{Kv} \beta 1.3$-specific probe detected the $3.8 \mathrm{~kb}$ transcript in the brain. Approximately $15 \mathrm{~kb}$ transcripts were detected in the DA, Ao, b-PA, UA, and UV. To document the presence of intact RNA in each lane, the same membrane was rehybridized with a porcine GAPDH probe (middle). Equal loading was demonstrated by ethidium bromide staining of the $28 \mathrm{~S}$ ribosomal RNA (bottom). $U A$, umbilical artery; $U V$, umbilical vein; $L V$, left ventricle; $R V$, right ventricle; $L A$, left atrium; $R A$, right atrium; $I S$, interventricular septum.

numbers: Kv1.2, DQ005441; Kv1.5, DQ005442; and kv9.3, DQ005443).

Northern blot analysis. The $\mathrm{Kv} \beta 1$ common probe hybridized to multiple bands (Fig. 4A). The $3.2 \mathrm{~kb}$ transcript was expressed highly in the DA and Ao, and faintly in the PA. The $3.8 \mathrm{~kb}$ transcript was expressed very weakly in the DA and Ao. Expression of the $3.2 \mathrm{~kb}$ transcript in the DA and Ao was confirmed by a $\operatorname{Kv} \beta 1.2$-specific probe (Fig. $4 B$ ). The $\operatorname{Kv} \beta 1.3$ specific probe was not hybridized to any of the DA, Ao, and PA as the $3.8 \mathrm{~kb}$ band. However, approximately $15 \mathrm{~kb}$ transcripts were detected in the DA and Ao (Fig. 4C). Although

Figure 3. Predicted amino acid sequences of Kv1.2, Kv1.5, and Kv9.3. Porcine Kv1.2 (A), Kv1.5 (B), Kv9.3 (C) amino acid sequences were deduced from the cloned nucleotide sequences, and aligned with amino acid sequences of corresponding $\mathrm{Kv} 1.2, \mathrm{Kv} 1.5$, and $\mathrm{Kv} 9.3$ from human, mouse, rat, respectively. Amino acid residues identical to the corresponding porcine $\mathrm{Kv}$ are shaded. $p$, porcine; $h$, human; $m$, mouse; $r$, rat.
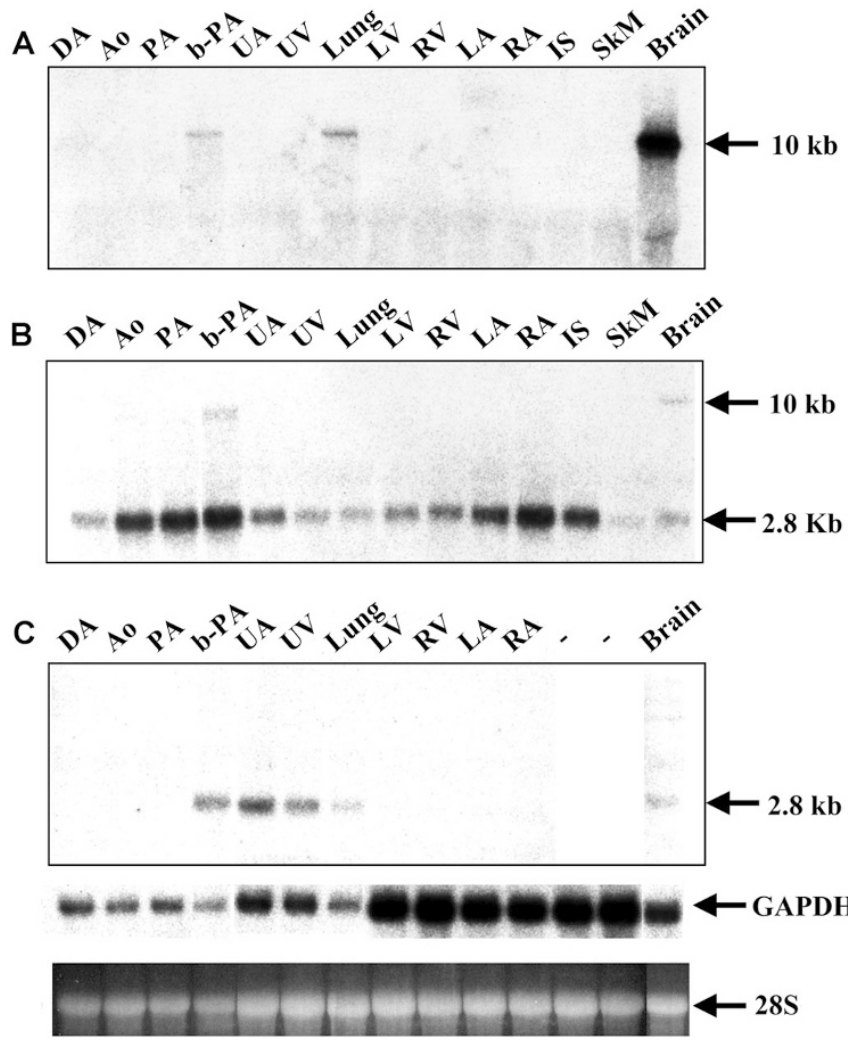

Figure 5. Northern blot analysis using (A) Kv1.2, (B) Kv1.5, and (C) Kv9.3 probes. (A) The Kv1.2 probe was hybridized to the approximately $10 \mathrm{~kb}$ transcripts in the brain, b-PA, and lung. (B) The Kv1.5 probe detected the 2.8 $\mathrm{kb}$ transcript abundantly in the Ao, PA, b-PA, LA, RA, and IS, but weakly in the DA, UA, UV, lung, LV, RV, SkM, and brain, and the $10 \mathrm{~kb}$ transcript in the b-PA and brain. (C) The Kv9.3 probe detected the $2.8 \mathrm{~kb}$ transcript in the b-PA, UA, and UV and weakly in the lung and brain. The same membrane was rehybridized with a porcine GAPDH probe (middle). Ethidium bromide staining of $28 \mathrm{~S}$ ribosomal RNA is shown below. Abbreviations are as in Figure 4.

we tried to detect $\operatorname{Kv} \beta 1.4$ using the $\operatorname{Kv} \beta 1$.4-specific probe, we could not obtain any signal on the Northern blot (data not shown).

Expression of Kv1.2 was not detected in the DA, Ao, or PA (Fig. 5A). Expression of the $2.8 \mathrm{~kb}$ transcripts was high in the Ao and PA, but low in the DA using the Kv1.5 probe (Fig. $5 B)$. Kv9.3 was not detected in the DA, Ao, or PA (Fig. $5 C$ ).

$\boldsymbol{q r}$-PCR. Expression of the $\mathrm{Kv} \beta 1.2$ transcript in the DA and Ao was high, and low in the PA (Fig. 6A). $\mathrm{Kv} \beta 1.3$ was detected very faintly in the DA and Ao. Expression of $\mathrm{Kv} \beta 1.4$ was not detected in the DA, Ao, or PA. Expression of $K v \beta 1.2$ dominated the other $\operatorname{Kv} \beta 1$ s among the blood vessels examined.

Kv1.2 was not detected in the DA, Ao, or PA (Fig. 6B). The expression of Kv1.5 in the Ao and PA was high, but relatively low in the DA. Kv2.1 was detected faintly in the Ao and PA. Expression of Kv9.3 was low in the DA, Ao, and PA. Expression of $\operatorname{Kv} \beta 1.2$ in the DA was higher than that of $\mathrm{Kv} 1.5$, and in the Ao and PA it was lower than that of Kv1.5 (Fig. 6C).

To estimate the potential contribution of EC in the DA, Ao, and PA samples, we used intracellular adhesion molecule 2 (ICAM-2) as EC-specific and SM22 $\alpha$ as SMC-specific 


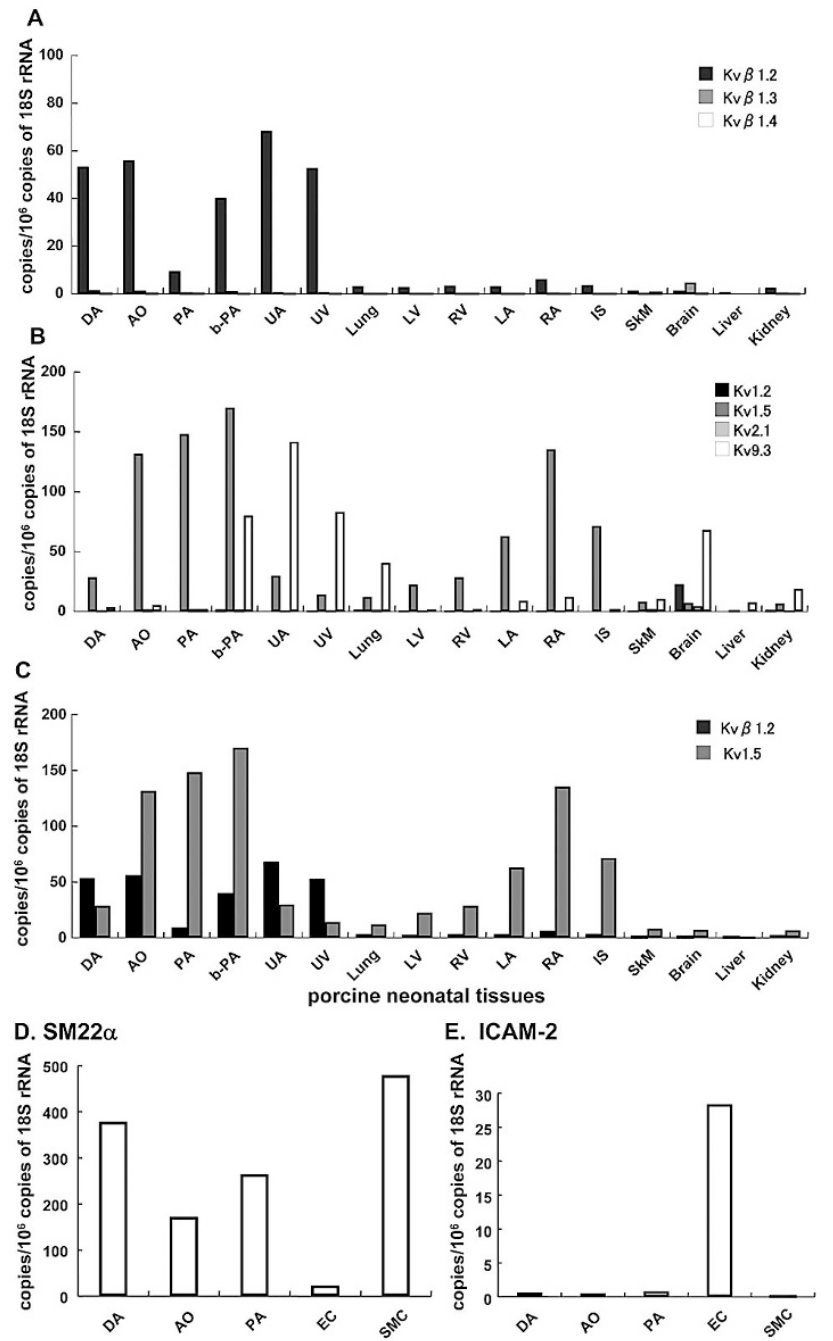

Figure 6. qr-PCR analysis of the expression of $\mathrm{Kv} \beta 1, \mathrm{Kv} 1.2, \mathrm{Kv} 1.5, \mathrm{Kv} 2.1$, and $\mathrm{Kv} 9.3$ transcripts. (A) Expression of the $\mathrm{Kv} \beta 1.2$ transcript was high in the DA, Ao, b-PA, UA, and UV and relatively low in the PA. Expression of $\mathrm{Kv} \beta 1.3$ was low in the brain and very low in the DA, Ao, b-PA, UA, and UV. Expression of $\mathrm{Kv} \beta 1.4$ was low in the SkM. Expression of $\mathrm{Kv} \beta 1.2$ dominated the other $\operatorname{Kv} \beta 1$ in the DA, Ao, b-PA, UA, and UV. (B) Expression of $\mathrm{Kv} 1.2$ was low in the brain and very low in the b-PA and lung. Expression of Kv1.5 was high in the Ao, PA, b-PA, LA, RA, and IS, and relatively low in the DA, UA, UV, Lung, LV, RV, SkM, brain, and kidney. Expression of $\mathrm{Kv} 2.1$ was low in the brain, and very low in the Ao, PA, b-PA, and SkM. Expression of Kv9.3 was high in the b-PA, UA, UV, lung, and brain, and low in the DA, Ao, LA, RA, SkM, liver, and kidney. (C) Comparison of the expression of $\operatorname{Kv} \beta 1.2$ and $\mathrm{Kv} 1.5$ mRNA Expression of $\mathrm{Kv} \beta 1.2$ in the DA, UA, and UV was higher than that of Kv1.5. The DA, Ao, and PA express high level of SM22 $\alpha(D)$, but low level of ICAM-2 $(E)$. Porcine aortic EC and $\mathrm{SMC}$ were used as positive controls. All mRNA levels were expressed as the copy number of the corresponding standard plasmid relative to that of the $18 \mathrm{~S}$ standard plasmid.

marker. Expression of SM22 $\alpha$ was high in the DA, Ao, and PA (Fig. $6 D$ ), but that of ICAM-2 was low (Fig. 6E).

Electrophysiology. A current activated by depolarization from a holding potential of $-60 \mathrm{mV}$ was evoked in the oocytes injected with cRNA encoding Kv1.5 (Fig. 7A) and Kv1.5/Kv $\beta 1.2$ (Fig. $7 B$ ). The outward currents evoked by a range of depolarizations from a holding potential of $-60 \mathrm{mV}$ to test potentials ranged from -40 to $+60 \mathrm{mV}$. A

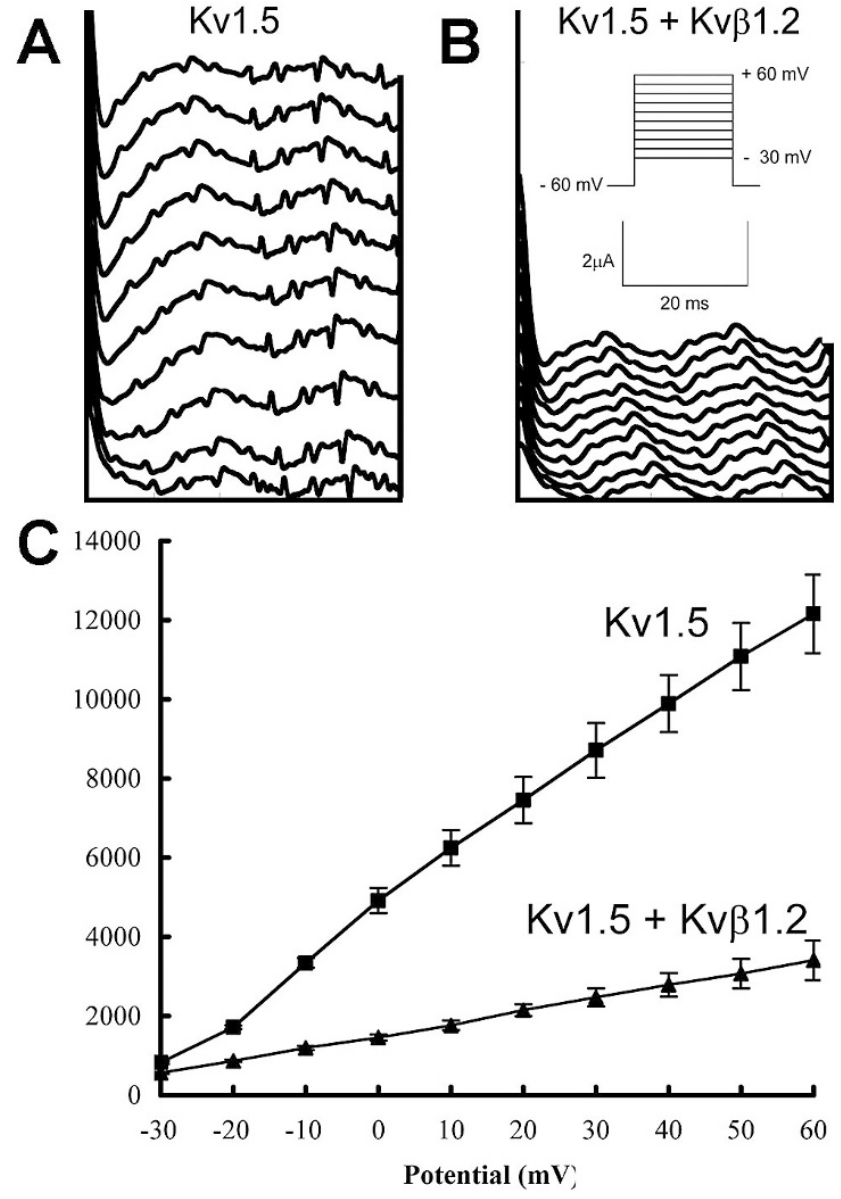

Figure 7. $\mathrm{K}^{+}$currents elicited from oocytes injected with porcine $\mathrm{Kv} 1.5$ cRNA alone $(A)$ or $\mathrm{Kv} 1.5$ and $\mathrm{Kv} \beta 1.2$ cRNA $(B) . \mathrm{K}^{+}$currents elicited from oocytes by steps from a holding potential of $-60 \mathrm{mV}$ to a range of test potentials from -30 to $+60 \mathrm{mV}$ in $10-\mathrm{mV}$ increments. Measurable current was seen with voltage steps to potentials positive of $-30 \mathrm{mV}$, which increased in a voltage-dependent manner. The current-voltage relationship for five oocytes is shown in $(C)$.

small current was detected at a test potential of $-40 \mathrm{mV}$, and this increased in a voltage-dependent manner with increasing depolarization. The I-V relationships of Kv1.5 and $\mathrm{Kv} 1.5 / \mathrm{Kv} \beta 1.2$ are shown in Figure $7 C$. When $\mathrm{Kv} \beta 1.2$ was co-expressed with Kv1.5, the amplitudes of the currents were decreased significantly.

\section{DISCUSSION}

In the present study, we demonstrated the first quantitative difference in expression of three porcine $\operatorname{Kv} \beta 1$ variants, $\mathrm{Kv} 1.2, \mathrm{Kv} 1.5$, Kv2.1, and Kv9.3 mRNA in various oxygensensitive blood vessels at birth. We used the adventitia and endothelial denuded DA, Ao, and PA as sample specimens. These expressed a high level of the SMC-specific marker but a low level of the EC-specific marker (Fig. 6, $D$ and $E$ ). Although some fibroblasts and EC may remain in the tissue, it is most likely that the specimens consist of the vascular SMC.

We found that expression of $\operatorname{Kv} \beta 1.2, \mathrm{Kv} 1.5$, and $\mathrm{Kv} 9.3$ were relatively high and characteristic in each blood vessel. Expression of $\mathrm{Kv} \beta 1.2$ in the DA was higher than that of 
$\mathrm{Kv} 1.5$. In contrast, expression of $\mathrm{Kv} \beta 1.2$ in the Ao, PA, and b-PA was lower than that of Kv1.5. We confirmed the inactivation property of $\operatorname{Kv} \beta 1.2$ against Kv1.5 using Xenopus laevis oocytes.

From these findings, $\mathrm{Kv} \beta 1.2$ in the DA might inactivate the Kv1.5 current effectively because of the higher expression of $\mathrm{Kv} \beta 1.2$. This inactivation might lead to SMC depolarization, opening of calcium channels, an increase in intracellular calcium, and vasoconstriction. In contrast, $\mathrm{Kv} \beta 1.2$ in the PA might inactivate the Kv1.5 current poorly, because of the much lower expression of $\operatorname{Kv} \beta 1.2$. The molecular basis for the differential electrophysiological characteristics in the PASMC and the DASMC might be due to a blood vessel-specific difference in expression of $\mathrm{Kv} 1.5$ and $\mathrm{Kv} \beta 1.2$.

$\operatorname{Kv} \beta$ belongs to the aldose-reductase superfamily of $\mathrm{NADPH}$, suggesting that it is an intracellular redox-sensing device for $\mathrm{Kv}$ channels (30). $\mathrm{Kv} \beta 1.2$ might play the role of sensing the cellular redox state and directly modifying the properties of the Kv1.5 subunit, depending upon the redox state, leading to the regulation of vascular constriction. The DA is known to constrict with an increase in oxygen at birth but the PA does not. The opposing response to oxygen might result from the difference in the relative expression levels of $\mathrm{Kv} 1.5$ and $\mathrm{Kv} \beta 1.2$.

The b-PA showed relatively high expression of Kv9.3 and a faint but detectable amount of Kv1.2 and Kv2.1 in addition to Kv1.5, suggesting acquisition of oxygen-sensitive properties under hypoxia and a physiologic distinction between the main PA and the b-PA.

Although expression of $\operatorname{Kv} \beta 1.2$ in heart has been described in adult specimens $(31,32)$, we did not detect $\operatorname{Kv} \beta 1.2$ in the porcine neonate heart. The diverse tissue distribution of $\mathrm{Kv} \beta 1.2$ may result from either differences in the developmental stage or species specificity.

The highly conserved sequences of $\operatorname{Kv} \beta 1.2$ and $\operatorname{Kv} \beta 1.3$ cDNA corresponding to human cDNA indicate that $\operatorname{Kv} \beta 1.2$ and $\operatorname{Kv} \beta 1.3$ might be generated through alternative splicing similar to human $\operatorname{Kv} \beta 1.2$ and $\operatorname{Kv} \beta 1.3$. The porcine $\operatorname{Kv} \beta 1$ gene might have a different splicing point compared with the corresponding human $\operatorname{Kv} \beta 1$ gene (Fig. 2). A high sequence similarity between the $5^{\prime}$-end of $\operatorname{Kv} \beta 1.4$ cDNA and the near $3^{\prime}$-end of the human intron 4 DNA was observed. Because the $\mathrm{Kv} \beta 1.4$ subunit lost its DNA sequences corresponding to human exons $1-4$ by this splicing, the putative start codon on exon 1 in ordinary $\mathrm{Kv} \beta 1$ genes was missing. Therefore, $\mathrm{Kv} \beta 1.4$ has the putative first initiating start codon on putative exon 8 , leading to a shorter predicted amino acid sequence.

$\operatorname{Kv} \beta 1.2$ and $\operatorname{Kv} \beta 1.3$ subunits both contain a " $\beta$ ball" in their $\mathrm{NH}_{2}$-terminal end with structural similarities to the " $\alpha$ ball" that works as an inactivating domain in A-type $\mathrm{Kv}$ channels. Therefore, $\operatorname{Kv} \beta 1.2$ and $\operatorname{Kv} \beta 1.3$ are expected to confer certain inactivation properties to the $\mathrm{Kv} \alpha$ subunits. In contrast, $\operatorname{Kv} \beta 1.4$ does not contain a " $\beta$ ball," suggesting that it is not able to inactivate the $\mathrm{Kv} \alpha$ subunits. Mouse $\mathrm{Kv} \beta 4.1$ also lacks a " $\beta$ ball" and enhances expression of $\mathrm{Kv} 2.2$ (25). Therefore, $\mathrm{Kv} \beta 1.4$ may have a role similar to that of $\operatorname{Kv} \beta 4.1$.
In this study, we demonstrated the quantitative difference in expression of $\mathrm{Kv} \beta 1$ variants, $\mathrm{Kv} 1.2, \mathrm{Kv} 1.5, \mathrm{Kv} 2.1$, and $\mathrm{Kv} 9.3$ mRNA in various oxygen-sensitive blood vessels at birth. Our results suggest that the molecular basis for the differential electrophysiological characteristics including opposing response to oxygen in the DA and the PA are partially due to diversity in expression of $\mathrm{Kv} 1.5$ and $\mathrm{Kv} \beta 1.2$ subunits. The high expression of $\operatorname{Kv} \beta 1.2$ and relatively low expression of Kv1.5 in the DA might be partially responsible for the ductal closure after birth. The potential involvement of $\operatorname{Kv} \beta 1.2$ in vascular constriction at birth as an intracellular redox-sensing device for $\mathrm{Kv}$ channels is one of the most interesting questions to be addressed in the future. Protein levels of the $\operatorname{Kv} \alpha$ and $\beta$ subunits as well as developmental changes in the expression of these proteins should be determined in a future study.

Acknowledgments. The authors thank Professor M. Lazdunski of Institut de Pharmacologie Moleculaire et Cellulaire (France) for providing rat Kv9.3 plasmid. We also thank B. Levene for English correction of the manuscript.

\section{REFERENCES}

1. Gilbert SF 1994 Developmental Biology. Sinauer Associates Inc., Sunderland, MA, pp 352-354

2. Kovalčík V 1963 The response of the isolated ductus arteriosus to oxygen and anoxia. J Physiol 169:185-197

3. Fay FS 1971 Guinea pig ductus arteriosus. I. Cellular and metabolic basis for oxygen sensitivity. Am J Physiol 221:470-479

4. Olley PM, Coceani F 1981 Prostaglandins and the ductus arteriosus. Annu Rev Med 32:375-385

5. Nakanishi T, Gu H, Hagiwara N, Momma K 1993 Mechanisms of oxygen-induced contraction of ductus arteriosus isolated from the fetal rabbit. Cir Res 72:1218-1228

6. Tristani-Firouzi M, Reeve HL, Tolarova S, Weir EK, Archer SL 1996 Oxygeninduced constriction of rabbit ductus arteriosus occurs via inhibition of a 4-aminopyridine-, voltage-sensitive potassium channel. J Clin Invest 98:1959-1965

7. Conforti L, Bodi I, Nisbet JW, Millhorn DE $2000 \mathrm{O}_{2}$-sensitive $\mathrm{K}^{+}$channels: role of the Kv1.2-subunit in mediating the hypoxic response. J Physiol 524:783-793

8. Thébaud B, Michelakis ED, Wu XC, Moudgil R, Kuzyk M, Dyck JR, Harry G, Hashimoto K, Haromy A, Rebeyka I, Archer SL 2004 Oxygen-sensitive Kv channel gene transfer confers oxygen responsiveness to preterm rabbit and remodeled human ductus arteriosus: Implications for infants with patent ductus arteriosus. Circulation 110:1372-1379

9. Hulme JT, Coppock EA, Felipe A, Martens JR, Tamkun MM 1999 Oxygen sensitivity of cloned voltage-gated $\mathrm{K}^{(+)}$channels expressed in the pulmonary vasculature. Circ Res 85:489-497

10. Osipenko ON, Tate RJ, Gurney AM 2000 Potential role for kv3.1b channels as oxygen sensors. Circ Res 86:534-540

11. Patel AJ, Lazdunski M, Honoré E 1997 Kv2.1/Kv9.3, a novel ATP-dependent delayed-rectifier $\mathrm{K}^{+}$channel in oxygen-sensitive pulmonary artery myocytes. EMBO J 16:6615-6625

12. Pérez-García MT, López-López JR, González C 1999 Kv $\beta 1.2$ subunit coexpression in HEK293 cells confers $\mathrm{O}_{2}$ sensitivity to kv4.2 but not to Shaker channels. J Gen Physiol 113:897-907

13. Pérez-García MT, Lopéz-Lopéz JR, Riesco AM, Hoppe UC, Marbán E, González C, John DC 2000 Viral gene transfer of dominant-negative Kv4 construct suppresses an $\mathrm{O}_{2}$-sensitive $\mathrm{K}^{+}$current in chemoreceptor cells. J Neurosci 20:5689-5695

14. Michelakis ED, Rebeyka I, Wu X, Nsair A, Thébaud B, Hashimoto K, Dyck JR, Haromy A, Harry G, Barr A, Archer SL $2002 \mathrm{O}_{2}$ sensing in the human ductus arteriosus: regulation of voltage-gated $\mathrm{K}^{+}$channels in smooth muscle cells by a mitochondrial redox sensor. Circ Res 91:478-486

15. MacKinnon R 1991 Determination of the subunit stoichiometry of a voltageactivated potassium channel. Nature 350:232-235

16. Heinemann S, Rettig J, Scott V, Parcej DN, Lorra C, Dolly J, Pongs O 1994 The inactivation behavior of voltage-gated K-channels may be determined by association of $\alpha$ - and $\beta$-subunits. J Physiol Paris 88:173-180

17. Rhodes KJ, Keilbaugh SA, Barrezueta NX, Lopez KL, Trimmer JS 1995 Association and colocalization of $\mathrm{K}^{+}$channel $\alpha$ - and $\beta$-subunit polypeptides in rat brain. J Neuroscience 15:5360-5371

18. Heinemann SH, Rettig J, Graack HR, Pongs O 1996 Functional characterization of Kv channel $\beta$-subunits from rat brain. J Physiol 493:625-633 
19. Xu J, Yu W, Wright JM, Raab RW, Li M 1998 Distinct functional stoichiometry of potassium channel $\beta$ subunits. Proc Natl Acad Sci U S A 95:1846-1851

20. Wang Z, Kiehn J, Yang Q, Brown AM, Wible BA 1996 Comparison of binding and block produced by alternatively spliced $\operatorname{Kv} \beta 1$ subunits. J Biol Chem 271:2831128317

21. Yang EK, Alvira MR, Levitan ES, Takimoto K $2001 \mathrm{Kv} \beta$ subunits increase expression of Kv4.3 channels by interacting with their C termini. J Biol Chem 276:4839-4844

22. England SK, Uebele VN, Kodali J, Bennett PB, Tamkun MM 1995 A novel $\mathrm{K}^{+}$ channel $\beta$-subunit $(\operatorname{hrv} \beta 1.3)$ is produced via alternative mRNA splicing. J Biol Chem 270:28531-28534

23. McCormack K, McCormack T, Tanouye M, Rudy B, Stühmer W 1995 Alternativesplicing of the human Shaker $\mathrm{K}^{+}$channel $\beta 1$ gene and functional expression of the $\beta 2$ gene product. FEBS Lett 370:32-36

24. Heinemann SH, Rettig J, Wunder F, Pongs O 1995 Molecular and functional characterization of a rat brain $\mathrm{Kv} \beta 3$ potassium channel subunit. FEBS Lett 377:383389

25. Fink M, Duprat F, Lesage F, Heurteaux C, Romey G, Barhanin J, Lazdunski M 1996 A new $\mathrm{K}^{+}$channel $\beta$ subunit to specifically enhance Kv2.2 (CDRK) expression. J Biol Chem 271:26341-26348
26. Chomczynski P, Sacchi N 1987 Single-step method of RNA isolation by acid guanidinium thiocyanate-phenol-chloroform extraction. Anal Biochem 162:156-159

27. Chomczynski P, Mackey K 1995 Short technical reports. Modification of the TRI reagent procedure for isolation of RNA from polysaccharide- and proteoglycan-rich sources. Biotechniques 19:942-945

28. Machida S, Noda S, Furutani Y, Takao A, Momma K, Matsuoka R 2000 Complete sequence and characterization of chick ventricular myosin heavy chain in the developing atria. Biochim Biophys Acta 1490:333-341

29. Overturf KE, Russell SN, Carl A, Vogalis F, Hart PJ, Hume JR, Sanders KM, Horowitz B 1994 Cloning and characterization of a Kv1.5 delayed rectifier K+ channel from vascular and visceral smooth muscles. Am J Physiol 267:C1231C1238

30. Gulbis JM, Mann S, MacKinnon R 1999 Structure of a voltage-dependent $\mathrm{K}^{+}$ channel $\beta$ subunit. Cell 97:943-952

31. England SK, Uebele VN, Shear H, Kodali J, Bennett PB, Tamkun MM 1995 Characterization of a voltage-gated $\mathrm{K}^{+}$channel $\beta$ subunit expressed in human heart. Proc Natl Acad Sci U S A 92:6309-6313

32. Morales MJ, Castellino RC, Crews AL, Rasmusson RL, Strauss HC 1995 A novel $\beta$ subunit increases rate of inactivation of specific voltage-gated potassium channel $\alpha$ subunits. J Biol Chem 270:6272-6277 